\section{Estudio comparativo de eficacia entre dos pañales para la prevención y tratamiento de la dermatitis por pañal}

Sáez de Ocariz-Gutiérrez $\mathrm{MM}^{1}$, Díaz-García $\mathrm{L}^{2}$, Contreras-Verduzco FA ${ }^{3}$, Narváez-Rosales $\mathrm{V}^{4}$, Solís-Ortiz $\mathrm{D}^{5}$, Greenawalt-Rodríguez $\mathrm{S}^{6}$, Espinosa-Padilla $\mathrm{S}^{3}$, Orozco-Covarrubias $\mathrm{L}^{1,7}$

\section{Resumen}

Una meta a alcanzar en los niños que usan pañal es mantener una piel intacta dentro del área cubierta por éste y así evitar la dermatitis por pañal.

OBJETIVO: comparar la eficacia de dos tipos de pañal para prevenir o tratar la dermatitis por pañal.

MATERIALES Y MÉTODOS: ensayo clínico aleatorizado en lactantes de 6 a 12 meses de edad, con o sin dermatitis por pañal, que utilizaron pañales Huggies UltraConfort ${ }^{\circledR}$ o BBTips ${ }^{\circledR}$. Se investigó la presencia o ausencia de dermatitis por pañal y la evaluación de la eficacia del pañal por el médico y el cuidador. El análisis estadístico incluyó análisis uni- y bivariado.

RESULTADOS: en el grupo sin dermatitis por pañal se evaluaron 68 lactantes con edad promedio de $8.54 \pm 2.09$ meses. Había tenido eventos previos de dermatitis por pañal $72 \%$. Catorce lactantes tuvieron dermatitis por pañal. La comparación entre ambos tipos de pañal demostró que BBTips ${ }^{\circledR}$ fue más eficaz que Huggies UItraConfort ${ }^{\circledR}$. En el grupo con dermatitis por pañal se evaluaron 66 pacientes con edad promedio de $8.65 \pm 2.4$ meses. Había tenido eventos previos de dermatitis por pañal $88 \%$. A los 15 días se habían resuelto $53 \%$ de las dermatitis por pañal y al mes $88 \%$. La comparación entre ambos tipos de pañal no demostró diferencias significativas en la eficacia.

CONCLUSIONES: en el aspecto preventivo BBTips ${ }^{\circledR}$ fue superior a Huggies UltraConfort ${ }^{\circledR}$. Ambos pañales tuvieron eficacia similar en el tratamiento de la dermatitis por pañal.

PALABRAS CLAVE: dermatitis por pañal, prevención, tratamiento.
${ }^{1}$ Servicio de Dermatología, Instituto Nacional de Pediatría.

2Departamento de Metodología de la investigación, Instituto Nacional de Pediatría.

${ }^{3}$ Pediatra e inmunólogo clínico y alergólogo, Hospital SEDNA.

${ }^{4}$ Pediatra y dermatóloga, Hospital Ángeles del Pedregal.

${ }^{5}$ Pediatra y dermatóloga, consultorio privado, Cuernavaca, Morelos.

${ }^{6}$ Pediatra.

${ }^{7}$ Pediatra y dermatóloga, Hospital Médica Sur.

Recibido: 3 de noviembre del 2015

Aceptado: 30 de junio del 2016

\section{Correspondencia}

Dra. María del Mar Sáez de Ocariz Gutiérrez mariadelmar71@prodigy.net.mx

Este artículo debe citarse como

Sáez de Ocariz-Gutiérrez MM, Díaz-García L, Contreras-Verduzco FA, Narváez-Rosales V, Solís-Ortiz D, Greenawalt-Rodríguez $S$ et al. Estudio comparativo de eficacia entre dos pañales para la prevención y tratamiento de la dermatitis por pañal. Acta Pediatr Mex. 2016;37(6):310-321. 


\section{Comparative efficacy trial between two types of diapers for the prevention and treatment of diaper dermatitis.}

Sáez de Ocariz-Gutiérrez $\mathrm{MM}^{1}$, Díaz-García $\mathrm{L}^{2}$, Contreras-Verduzco $\mathrm{FA}^{3}$, Narváez-Rosales $\mathrm{V}^{4}$, Solís-Ortiz $\mathrm{D}^{5}$, Greenawalt-Rodríguez $\mathrm{S}^{6}$, Espinosa-Padilla $\mathrm{S}^{3}$, Orozco-Covarrubias $\mathrm{L}^{1,7}$

\begin{abstract}
BACKGROUND: To maintain the integrity of the skin in the area covered by a diaper is a goal in order to avoid the development of diaper dermatitis (DD).
\end{abstract}

OBJECTIVE: To compare the efficacy between two types of diapers to prevent or treat DD.

PATIENTS AND METHODS: Clinical randomized trial in which infants from 6 to 12 months of age, with or without DD, who used either Huggies UltraConfort ${ }^{\mathrm{TM}}$ or BBTips ${ }^{\mathrm{TM}}$, were included. The presence or absence of DD and the diapers' efficacy evaluation by the physician and the keeper were documented. Statistical analysis included uni and bivariate analyses.

RESULTS: In the group without DD, 68 infants with a mean age of $8.54 \pm 2.09$ months were evaluated. Seventy-two percent had had previous events of DD. Fourteen infants developed DD. The comparison between both types of diaper showed that BBTips TM had a superior efficacy over Huggies UltraConfort ${ }^{\mathrm{TM}}$. In the group with DD, 66 patients with a mean age of $8.65 \pm 2.4$ months were evaluated. Eighty-eight percent had had previous events of DD. Fifty-three percent of the DD had resolved by day 15 , and $88 \%$ by day 30 . The comparison between both types of diapers did not show significant differences in efficacy.

CONCLUSIONS: BBTips ${ }^{\mathrm{TM}}$ was superior to Huggies UltraConfort ${ }^{\mathrm{TM}}$ for the prevention of DD. Both types of diapers had a similar efficacy for the treatment of DD.

KEYWORDS: diaper dermatitis; prevention; treatment
${ }^{1}$ Servicio de Dermatología, Instituto Nacional de Pediatría.

2Departamento de Metodología de la investigación, Instituto Nacional de Pediatría.

${ }^{3}$ Pediatra e inmunólogo clínico y alergólogo, Hospital SEDNA.

${ }^{4}$ Pediatra y dermatóloga, Hospital Ángeles del Pedregal.

${ }^{5}$ Pediatra y dermatóloga, consultorio privado, Cuernavaca, Morelos.

${ }^{6}$ Pediatra.

${ }^{7}$ Pediatra y dermatóloga, Hospital Médica Sur.

\section{Correspondence}

Dra. María del Mar Sáez de Ocariz Gutiérrez mariadelmar71@prodigy.net.mx

\section{INTRODUCCIÓN}

El uso de pañal es una forma efectiva y conveniente de mantener contenidas las excretas de los lactantes. Sin embargo, la piel no fue diseñada para operar en forma continua en el ambiente resultante, caracterizado por humedad constante y oclusión. Ambas elevan el 
coeficiente de fricción de la piel bajo el pañal, comprometiendo su integridad y su capacidad de responder a irritantes secundarios; $;^{1-3}$ por lo que con mucha frecuencia la piel es incapaz de defenderse de esta "agresión" ${ }^{4}$ dando como resultado una "rozadura" o dermatitis por pañal irritativa. $^{5}$

La dermatitis por pañal irritativa es la principal causa de consulta dermatológica en lactantes y preescolares, con una prevalencia entre 40 y $50 \%$ de los pacientes en estos grupos etarios. ${ }^{6-9}$ Aunque sólo 5\% de las dermatitis por pañal se consideran graves ${ }^{10}$ las formas leves y moderadas revisten importancia para los lactantes, sus padres, cuidadores, pediatras o médicos de primer contacto.

El pañal ideal no existe, pues aún se observa una elevada prevalencia de dermatitis por pañal en los lactantes entre los 6 y 12 meses de edad; ${ }^{5}$ sin embargo, los pañales desechables y su composición han mejorado significativamente en los últimos años, así como la investigación respecto a su elaboración. ${ }^{11}$ El uso de pañales desechables con materiales superabsorbentes o con gel, ${ }^{12-15} \mathrm{o}$ incluso aquéllos que pueden liberar fórmulas con óxido de zinc/petrolato, ${ }^{14,16}$ muestran una menor incidencia y gravedad de dermatitis por pañal.

Mantener una piel intacta dentro del área cubierta por el pañal sigue siendo una meta a alcanzar; ${ }^{17}$ por ello se justifica continuar buscando opciones de pañales desechables que se acerquen más al ideal y se reduzcan la frecuencia y gravedad de la dermatitis por pañal en los lactantes en riesgo.

\section{OBJETIVO}

Comparar la eficacia de los pañales BBTips ${ }^{\circledR}$ versus Huggies UltraConfort ${ }^{\circledR}$ para prevenir o tratar dermatitis por pañal en lactantes de 6 a
12 meses de edad, desde el punto de vista del médico tratante y del cuidador.

\section{MATERIALES Y MÉTODOS}

Ensayo clínico de eficacia simple, ciego (para quién realizó el análisis estadístico), en el que se incluyeron lactantes de 6 a 12 meses de edad sin dermatitis por pañal irritativa y con dermatitis por pañal irritativa (con o sin sobreinfección por Cándida albicans) atendidos en un período de 6 meses en la consulta privada de pediatras y dermatólogos pediatras del Distrito Federal (FACV, VNR, SGR, SEP, LOC) y Cuernavaca (DOS), cuyos padres aceptaron participar en el estudio y firmaron una carta de consentimiento. En el grupo 1 (sin dermatitis por pañal) se excluyeron los lactantes que presentaran alguna dermatosis en el área del pañal y en el grupo 2 (con dermatitis por pañal) se excluyeron los lactantes que hubieran recibido tratamiento con esteroides tópicos en los 15 días previos a su inclusión en el estudio. En ambos grupos se eliminaron a los lactantes que no acudieron a las visitas de seguimiento. El procedimiento general de estudio se muestra en la Figura 1.

Los pediatras y los dermatólogos pediatras fueron estandarizados para la aplicación de la escala de gravedad de la dermatitis por pañal (Cuadro 1) $)^{18}$ utilizada en la evaluación de los pacientes del grupo 2 .

Los cuidados generales para el área que cubre el pañal en pacientes del grupo 1 (sin dermatitis por pañal) incluyeron: cambio de pañal cada 4 horas o ante la presencia de orina o heces; aseo del área con agua y jabones suaves o torundas humedecidas en agua (sin utilizar toallitas húmedas desechables); secado gentil y orear el área por algunos minutos y aplicación de vaselina sólida en cada cambio de pañal.

El tratamiento convencional de la dermatitis por pañal (grupo 2) incluyó: cambio de pañal cada 


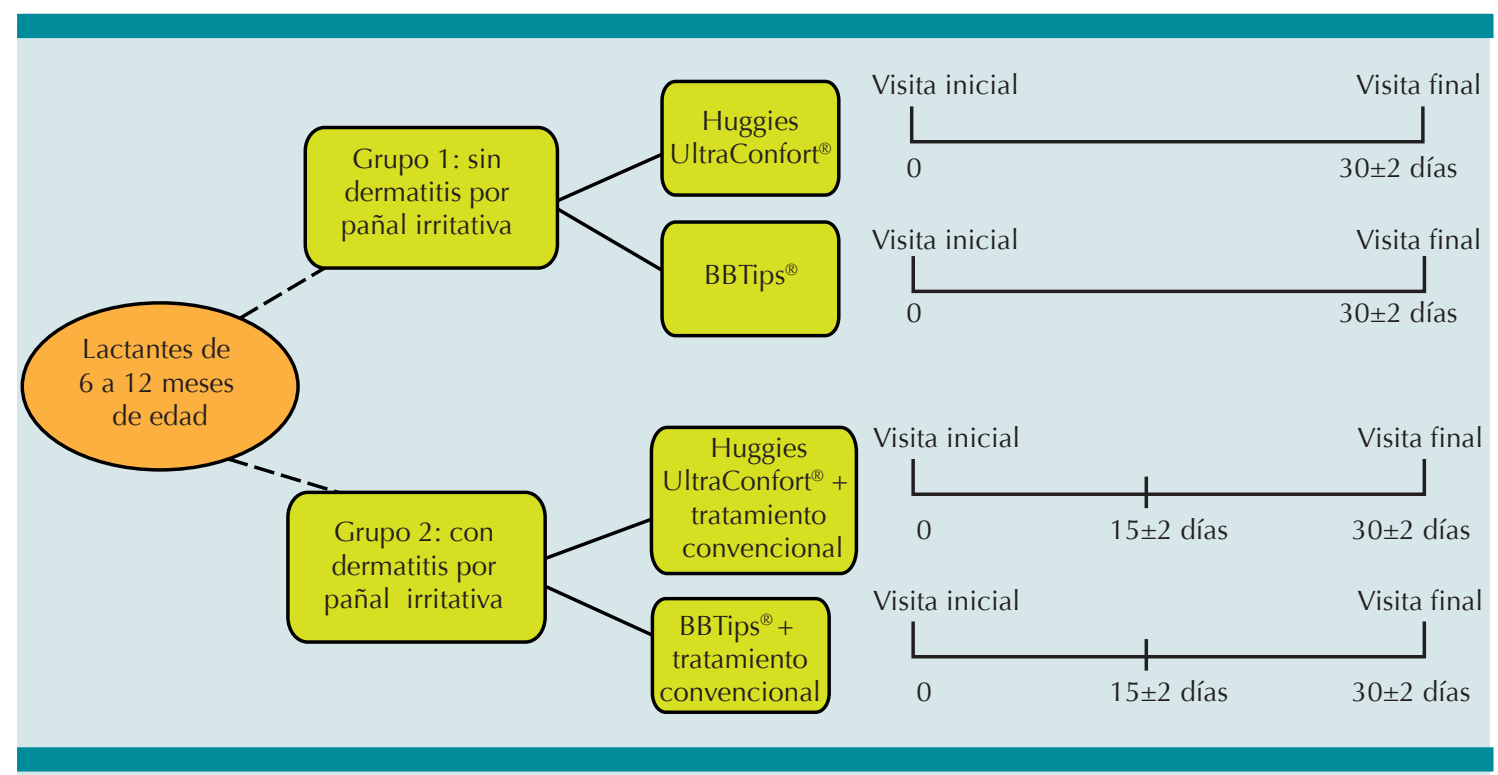

Figura 1. Procedimiento general del estudio.

Cuadro 1. Escala de gravedad de la dermatitis por pañal

0 Piel sana

1 Eritema mínimo en toda el área del pañal o en parche en áreas localizadas; leve irritación

2 Eritema en toda el área del pañal o en áreas localizadas con pápulas eritematosas; irritación moderada

3 Eritema de mayor intensidad en forma difusa asociado con pápulas eritematosas; irritación considerable

4 Eritema intenso, con o sin exudado, en un patrón difuso asociado con pápulas, pústulas o exulceraciones; irritación extrema

5 Eritema extremo que afecta toda el área del pañal asociado con exudado, pápulas, pústulas y erosiones

1 y 2 = leve; 3 = moderado; 4 = moderado-grave; 5 = grave.

Modificado de Davis JA, Leyden JJ, Grove GL, Raynor WJ. Comparison of disposable diapers with fluff absorbent and fluff plus absorbent polymers: Effects on skin hydration, skin pH, and diaper dermatitis. Pediatric Dermatol 1989;6(2):102-08. ${ }^{18}$

4 horas o ante la presencia de orina o heces; aseo del área con agua y jabones suaves o con torundas humedecidas en agua (sin utilizar toallitas húmedas desechables); secado gentil y orear el área por algunos minutos y aplicación de un ungüento de barrera, elegido a discreción del médico tratante, en cada cambio de pañal. En caso de existir candidiasis agregada se prescribieron pomadas con clioquinol, nistatina o algún otro antimicótico tópico, elegido por el médico tratante, dos veces al día.

Los dos tipos de pañal evaluados en este estudio pertenecen al grupo de pañales con absorbentes en gel y ambos están compuestos por una cubierta interna adicionada con manzanilla, aloe y vitamina E; una cubierta externa respirable y cintas de velcro con elástico. Tanto BBTips ${ }^{\circledR}$ Como 
Huggies UltraConfort ${ }^{\circledR}$ tienen características muy semejantes en cuanto a su capacidad y velocidad de absorción, su porcentaje de distribución, así como cintas de velcro con elástico, elástico en la espalda y corte con elástico ergonómico, según el "Estudio de Calidad. Pañales Desechables. La Misión Antiescurrimiento" publicado en la Revista del Consumidor en 2011. ${ }^{19}$

Se calculó el tamaño de muestra con la fórmula de diferencias entre dos proporciones ${ }^{20}$ considerando diferentes estudios sobre la prevalencia de la dermatitis por pañal, ${ }^{6-9}$ resultando un total de 140 pacientes, 70 pacientes por grupo de estudio. En el grupo 1 se consideraron lactantes sin dermatitis por pañal y en el grupo 2 pacientes con dermatitis por pañal al momento de su inclusión en el estudio. En cada grupo, 35 lactantes fueron asignados a Huggies UltraConfort ${ }^{\circledR}$ y 35 lactantes a BBTips ${ }^{\circledR}$; se realizó asignación aleatoria del pañal a ser prescrito mediante bloques balanceados de tamaño 10 .

Las variables consideradas en el grupo 1 fueron: sexo, edad al diagnóstico, cuidador principal, eventos previos de dermatitis por pañal, frecuencia basal del cambio de pañal, desarrollo de dermatitis por pañal durante el estudio, seguimiento de las indicaciones por el cuidador, eficacia del pañal en la prevención de la dermatitis por pañal, según los criterios del médico y del cuidador, y si se volvería a utilizar en el futuro el pañal prescrito.

Las variables consideradas en el grupo 2 fueron: sexo, edad al diagnóstico, cuidador principal, eventos previos de dermatitis por pañal; frecuencia basal del cambio de pañal, presencia de candidiasis agregada en las visitas basal, a los 15 y a los 30 días; seguimiento de las indicaciones por el cuidador, resolución de la dermatitis por pañal a los 15 y 30 días, eficacia del pañal en el tratamiento de la dermatitis por pañal según los criterios del médico y del cuidador y si se volvería a utilizar en el futuro el pañal prescrito.

La evaluación estadística incluyó análisis univariado con frecuencias o proporciones para las variables cualitativas, medidas de tendencia central y dispersión para las variables cualitativas. En el análisis bivariado se utilizaron $\chi^{2}$ y $t$ de Student.

El protocolo fue aprobado por el Comité de Ética en Investigación del Hospital Ángeles del Pedregal, con número de registro 2381.

\section{RESULTADOS}

De los 138 pacientes 70 estaban en el grupo 1, 68 en el grupo 2 y se eliminaron 4 pacientes por pérdida de seguimiento: 2 del grupo 1 y 2 del grupo 2, resultando 68 pacientes en el grupo 1 y 66 pacientes en el grupo 2 (Figura 2). Las características basales de la población en estudio fueron muy semejantes entre sí (Cuadro 2) excepto por

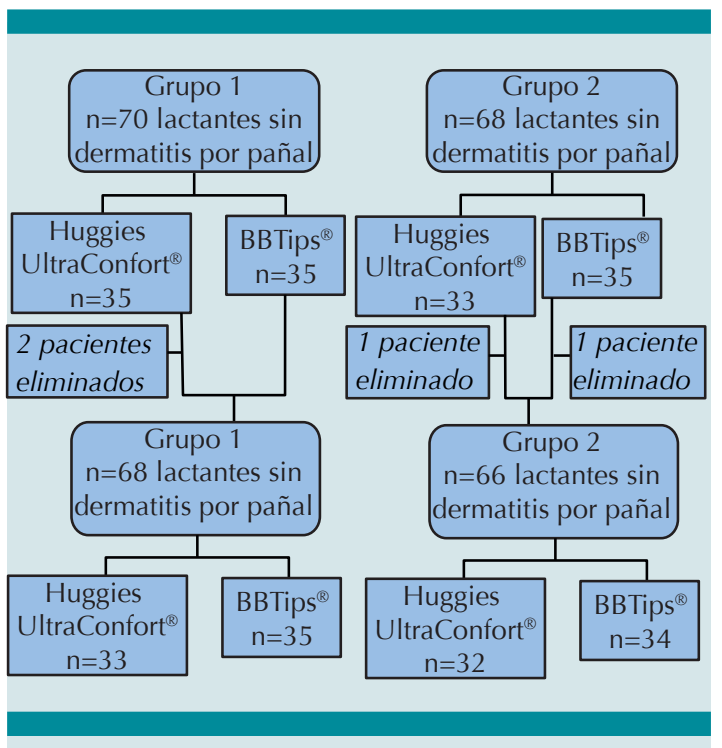

Figura 2. Captación de pacientes, aleatorización, asignación de pañal, inclusión y eliminación de pacientes a lo largo del estudio, para cada uno de los grupos. 
Cuadro 2. Características basales de ambos grupos

\begin{tabular}{|l|c|c|}
\hline Variable & $\begin{array}{c}\text { Grupo sin dermatitis por pañal } \\
\mathbf{n = 6 8}\end{array}$ & $\begin{array}{c}\text { Grupo con dermatitis por pañal } \\
\mathbf{n = 6 6}\end{array}$ \\
\hline $\begin{array}{l}\text { Sexo }(\mathrm{n}[\%]) \\
\quad \text { Masculino }\end{array}$ & $29[42.6]$ & $29[43.9]$ \\
$\quad$ Femenino & $39[57.4]$ & $37[56.1]$ \\
\hline Edad en meses $(\boldsymbol{\mu} \mathrm{DE})$ & $8.5 \pm 2.09$ & $8.65 \pm 2.39$ \\
\hline $\begin{array}{l}\text { Antecedente de evento previo de dermatitis por } \\
\text { pañal }{ }^{*}(\mathrm{n}[\%])\end{array}$ & $49[72.1]$ & $58[87.9]$ \\
\hline Madre como principal cuidadora $(\mathrm{n}[\%])$ & $59[86.6]$ & $57[86.4]$
\end{tabular}

${ }^{*} \chi^{2}=5.2 \mathrm{p} 0<0.019$

el antecedente previo de dermatitis por pañal, que fue mayor en el grupo $2(p=0.019)$.

\section{Grupo 1: lactantes sin dermatitis por pañal}

De los 70 lactantes, 68 concluyeron el estudio $(97.1 \%)$ y 2 fueron eliminados $(2.9 \%)$ por pérdida de seguimiento, a pesar de repetidas llamadas telefónicas. Los 2 casos perdidos no tenían antecedentes previos de dermatitis por pañal y acudieron a consulta inicial como control de niño sano.

De los 68 niños sin dermatitis por pañal 39 (57.4\%) fueron del sexo femenino y la edad promedio fue de $8.54 \pm 2.09$ meses. El motivo principal de consulta de los lactantes fue control del niño sano en $58.8 \%$ de los casos, seguido por control de dermatitis atópica en $8.8 \%$.

Mediante método aleatorio se asignó a 50\% de los lactantes a cada grupo de pañal, pero al ser eliminados 2 lactantes quedaron 33 niños (48.5\%) que usaron Huggies Ultra-Confort ${ }^{\circledR}$ y 35 niños $(51.5 \%)$ que usaron BBTips ${ }^{\circledR}$. Treinta y seis lactantes $(52.9 \%)$ fueron atendidos por dermatólogos pediatras y 32 (47.1\%) por pediatras.

De los 68 pacientes $72.1 \%$ había tenido eventos previos de dermatitis por pañal. Los episodios previos variaban de $1(26.5 \%), 2(16.2 \%), 3$ (8.8\%), 4 (11.8\%), 5 (4.4\%) hasta 6 o más (5.9\%) veces, con un promedio de $1.84 \pm 1.81$.

En el momento de la consulta el cuidador principal del niño fue la madre en $86.6 \%$ de los casos; el padre en $8.8 \%$ de los casos y otro familiar (tíos o abuelos) en $4.4 \%$ de los casos. La frecuencia de cambio de pañal que se informó fue cada 4 horas en $52.9 \%$, antes de 4 horas en $35.3 \%$ y por lo menos una hora después en $11.8 \%$ de los casos, con un promedio de $3.77 \pm 0.84$ horas.

En forma general 14 de los 68 niños (20.6\%) desarrollaron dermatitis por pañal a lo largo del estudio; en 93.3\% se trató de una afección leve y en un paciente fue moderada.

\section{Comparación entre ambos pañales}

El Cuadro 3 muestra las características comparadas entre ambos grupos. No hubo diferencias significativas en eventos previos de dermatitis por pañal, en el desarrollo de ésta a lo largo del estudio, ni en si se volvería a utilizar en el futuro el pañal prescrito, entre ambos grupos.

Todos los casos de dermatitis por pañal (14) a lo largo del estudio fueron leves, con excepción 
Cuadro 3. Comparación entre ambos pañales en el grupo de lactantes sin dermatitis por pañal

\begin{tabular}{|c|c|c|c|}
\hline Variable & $\begin{array}{l}\text { Huggies UltraConfort } \\
\qquad \mathrm{n}=32\end{array}$ & $\begin{array}{c}\text { BBTIps }^{\circledR} \\
n=34\end{array}$ & $p$ \\
\hline Edad en meses ( $\mu \mathrm{DE})$ & $8.58 \pm 2.17$ & $8.57 \pm 2.06$ & $0.93 *$ \\
\hline Eventos previos de dermatitis por pañal (n [\%]) & $22[66.7]$ & $27[77.1]$ & $0.34-$ \\
\hline Eventos previos de dermatitis por pañal ( $\mu$ D.E.) & $1.79 \pm 2.01$ & $2.00 \pm 1.61$ & $0.63^{*}$ \\
\hline Desarrollo de dermatitis por pañal durante el estudio (n [\%]) & 10 [30.3] & 5 [14.3] & $0.11 * *$ \\
\hline $\begin{array}{l}\text { El pañal fue eficaz para prevenir el desarrollo de } \\
\text { dermatitis por pañal según el médico }(\mathrm{n}[\%])\end{array}$ & 23 [69.7] & $31[88.6]$ & $0.04^{* *}$ \\
\hline $\begin{array}{l}\text { El pañal fue eficaz para prevenir el desarrollo de } \\
\text { dermatitis por pañal según el cuidador ( }[\text { [\%]) }\end{array}$ & $25[75.8]$ & 33 [94.3] & $0.03^{* *}$ \\
\hline El cuidador volvería a usar el pañal prescrito (n [\%]) & $24[72.7]$ & $31[88.6]$ & $0.09 * *$ \\
\hline
\end{tabular}

${ }^{*}$ t de Student; ** $\chi^{2}$

de uno que fue moderado en un lactante que usó BBTips ${ }^{\circledR}$.

Según el criterio médico el pañal no fue eficaz para prevenir el desarrollo de dermatitis por pañal en 14 niños. Diez de ellos usaron Huggies Ultra-Confort ${ }^{\circledR}$, en tanto que 4 usaron BBTips ${ }^{\circledR}$, la diferencia fue estadísticamente significativa $(p=0.045)$.

Según el criterio del cuidador el pañal no fue eficaz para prevenir el desarrollo de dermatitis en 9 niños. Siete de ellos usaron Huggiesa ${ }^{\hat{a}}$ UltraConfort $^{\circledR}$ y los 2 restantes BBTips ${ }^{\circledR}$, la diferencia fue estadísticamente significativa ( $p=0.031)$.

\section{Grupo 2: lactantes con dermatitis por pañal}

De los 68 pacientes se eliminaron 2 por falta de seguimiento. Treinta y siete $(56.1 \%)$ del sexo femenino y la edad promedio fue de $8.65 \pm 2.40$ meses. Después de la eliminación de 2 lactantes, uno con cada pañal, 32 lactantes (48.5\%) recibieron Huggies UltraConfort ${ }^{\circledR}$ y 34 (51.5\%) BBTips $^{\circledR}$. En cuanto a la atención de pacientes $44(66.7 \%)$ fueron atendidos por pediatras y 22 (33.3\%) por dermatólogos pediatras.
De los 66 pacientes $87.9 \%$ había tenido eventos previos de dermatitis por pañal que variaban de $1(15.2 \%), 2$ (15.2\%), 3 (16.7\%), 4 (16.7\%), $5(7.6 \%)$ hasta 6 o más $(16.7 \%)$ veces, con un promedio de $3.00 \pm 1.97$.

\section{Evaluación inicial de la dermatitis por pañal}

La gravedad de la dermatitis por pañal evaluada por el médico fue de $65.1 \%$ (43) de los niños de forma leve; $28.8 \%$ (19) de forma moderada; $4.5 \%$ (3) de forma moderada-grave y $1.5 \%$ (1) de forma grave. En 15.2\% de los casos (10) la dermatitis por pañal se asoció con Cándida.

En todos los pacientes se dieron indicaciones de tratamiento convencional. Las pastas prescritas para cada cambio de pañal se muestran en el Cuadro 4.

Los niños con Cándida (15\%) recibieron un antimicótico tópico: itraconazol en 8 casos y ciclopirox en 2 casos.

El cuidador de los pacientes fue la madre en $86.4 \%$ de los casos; el padre en $12.1 \%$ y algún otro cuidador (tíos o abuelos) en $1.5 \%$ de los 
Cuadro 4. Pastas del pañal prescritas para el tratamiento de la dermatitis por pañal

\section{Tipo de pasta}

Óxido de zinc

Petrolato puro

$11(16.7)$

Óxido de zinc + petrolato puro

$12(18.2)$

Dexpantenol

$20(30.3)$

Óxido de zinc + calamina

$14(21.2)$

Lanolina + óxido de zinc

$4(6.1)$

Agua termal + sulfato de cobre + sulfato de zinc 2 (3.0)

+ óxido de zinc + sucralfato

Agua de rosas + óxido de zinc + glicerina

$1(1.5)$

casos. La frecuencia de cambio de pañal fue de cada 4 horas en $40.9 \%$, antes de 4 horas en $49.9 \%$ y por lo menos una hora después en $9.1 \%$ de los casos, con un promedio de $3.42 \pm 0.89$ horas.

La dermatitis por pañal, de acuerdo con el cuidador del paciente, fue leve en 36 pacientes $(54.5 \%)$, moderada en $14(25.8 \%)$, moderadagrave en $8(12.1 \%)$ y grave en $5(7.6 \%)$.

Evaluación de la dermatitis por pañal a los 15 días de tratamiento

A los 15 días de tratamiento la dermatitis por pañal se resolvió en 53\% de los pacientes. En quiénes persistió fueron, en su mayoría (90.3\%), formas leves y en menor grado moderadas $(9.7 \%)$. En $4.5 \%$ de los casos se asoció con Cándida.

En todos los pacientes en quienes se resolvió la dermatitis por pañal se indicó continuar con cuidados generales para el área que cubre el pañal. En todos los pacientes en quiénes persistió la dermatitis por pañal se indicó el mismo tratamiento prescrito en la visita inicial. A los 3 niños que presentaron Cándida además se les prescribió un antimicótico tópico: itraconazol en 2 pacientes y ciclopirox en el otro.
Todos los cuidadores refirieron haber seguido las indicaciones. Según el criterio del cuidador la dermatitis por pañal se resolvió en 53\% de los pacientes $y$, en quienes persistió, fueron formas leves en la mayoría de los casos $(77.41 \%)$, moderadas en $19.35 \%$ y graves en $3.22 \%$.

\section{Evaluación final}

Al mes de tratamiento la dermatitis por pañal se resolvió en $87.9 \%$ de los casos; en aquéllos en quiénes persistió fueron de forma leve (87.5\%) y en menor grado moderada (12.5\%). En ningún caso se observó asociación con Cándida.

El pañal en general fue considerado por el médico como eficaz para el tratamiento de la dermatitis por pañal en 58 pacientes $(87.9 \%)$ de los casos.

El 95.5\% de los cuidadores refirió haber seguido las indicaciones. En los 3 casos en que no se siguieron por haber utilizado toallas húmedas. Según el criterio del cuidador la dermatitis por pañal se resolvió en $87.9 \%$ de los casos y persistió en $12.1 \%$; fue leve ( $87.5 \%$ ) en la mayoría de los casos y de moderada a grave en los restantes.

El 87.9.\% de los cuidadores consideró al pañal eficaz para el tratamiento de la dermatitis por pañal y $93.9 \%$ de ellos refirió que los seguiría utilizando en el futuro.

\section{Comparación entre ambos pañales}

En el Cuadro 5 se pueden observar las características que fueron comparadas entre ambos grupos. Las variables que mostraron diferencias significativas fueron la cantidad de eventos previos y el promedio de eventos previos de dermatitis por pañal, que fue mayor en los pacientes que recibieron Huggies UltraConfort ${ }^{\circledR}$. 
Cuadro 5. Comparación entre ambos pañales en el grupo de lactantes con dermatitis por pañal

\begin{tabular}{|c|c|c|c|}
\hline Variable & $\begin{array}{l}\text { Huggies UltraConfort }{ }^{\circledR} \\
\qquad n=32\end{array}$ & $\begin{array}{c}\text { BBTips }^{\circledR} \\
\mathrm{n}=34\end{array}$ & $p$ \\
\hline Edad en meses ( $\mu$ D.E.) & $9.00 \pm 2.45$ & $8.32 \pm 2.33$ & $0.255^{*}$ \\
\hline Eventos previos de dermatitis por pañal (n [\%]) & $32[100]$ & $26[76.5]$ & $0.003^{* *}$ \\
\hline Eventos previos de dermatitis por pañal ( $\mu$ D.E.) & $3.56 \pm 1.66$ & $2.47 \pm 2.11$ & $0.023^{*}$ \\
\hline Frecuencia basal de cambio de pañal ( $\mu$ D.E.) & $3.47 \pm 0.80$ & $3.38 \pm 0.99$ & $0.698^{*}$ \\
\hline Candida en visita inicial (n [\%]) & $4[12.5]$ & $6[17.6]$ & $0.560^{* *}$ \\
\hline Resolución de la dermatitis por pañal al día 15 (n [\%]) & 15 [46.9] & 20 [58.8] & 0.331 \\
\hline Candida en visita intermedia (n [\%]) & $1[3.1]$ & $2[5.9]$ & $0.591^{* *}$ \\
\hline Seguimiento de indicaciones por el cuidador, los primeros 15 días (n [\%]) & $32[100]$ & $34[100]$ & - \\
\hline Resolución de la dermatitis por pañal al día 30 (n [\%]) & $28[87.5]$ & $30[88.2]$ & $0.927 * *$ \\
\hline Candida en visita final ( $\mathrm{n}[\%]$ ) & $0[0]$ & $0[0]$ & - \\
\hline $\begin{array}{l}\text { Seguimiento de indicaciones por el cuidador, los siguientes } 15 \text { días } \\
\text { (n [\%]) }\end{array}$ & 29 [90.6] & $34[100]$ & $0.069 * *$ \\
\hline $\begin{array}{l}\text { El pañal fue eficaz en el tratamiento de la dermatitis por pañal según } \\
\text { el médico (n [\%]) }\end{array}$ & 28 [87.5] & $30[88.2]$ & $0.927^{* *}$ \\
\hline $\begin{array}{l}\text { El pañal fue eficaz en el tratamiento de la dermatitis por pañal según } \\
\text { el cuidador ( } n[\%])\end{array}$ & $27[84.4]$ & $31[91.2]$ & $0.397 * *$ \\
\hline El cuidador volvería a usar el pañal prescrito (n [\%]) & 31 [96.9] & $31[91.2]$ & $0.332 * *$ \\
\hline
\end{tabular}

${ }^{*}$ t de Student; ${ }^{* *} \chi^{2}$

En cuanto a las demás variables, en particular la eficacia del pañal en el tratamiento de la dermatitis por pañal, evaluada tanto por el médico como por el cuidador, fue muy similar en ambos grupos.

\section{DISCUSIÓN}

La dermatitis por pañal irritativa es una enfermedad de etiología multifactorial caracterizada por inflamación en el área cubierta por el pañal. Es causada por una combinación de efectos primariamente irritativos dentro de los que se incluyen el uso del pañal, incremento del pH cutáneo, exposición prolongada a la humedad e irritantes como la orina y las heces; ${ }^{21,22}$ se caracteriza por eritema confluente, pápulas eritematosas, edema y descamación en las áreas convexas de las nalgas, las ingles, el pubis y el escroto o los labios mayores. ${ }^{3}$ Se pueden agregar agentes infecciosos como Candida albicans que prolifera en la piel cuando hay suficiente humedad y calor, lo cual genera mayor inflamación acompañada de pápulas y pústulas satélite. ${ }^{23}$

Como la dermatitis por pañal irritativa tiene su máxima presentación entre los 6 y 12 meses de edad (aunque puede continuar hasta que se elimina el uso del pañal $)^{2}$ en este estudio se seleccionó precisamente la población de mayor riesgo para evaluar la eficacia de ambos pañales para prevenir o tratar la dermatitis por pañal irritativa.

La eficacia en la prevención de la dermatitis por pañal se evaluó en lactantes sin ella al momento de la inclusión al estudio, con una pérdida de seguimiento en $2.9 \%$ de los casos, que es menor al $10 \%$ esperado y considerado dentro del cálculo de la muestra. ${ }^{20}$ Como es conocido que el 
desarrollo de la dermatitis por pañal no se debe únicamente al uso del pañal, sino que influyen otros factores como el incremento del $\mathrm{pH}$, la humedad y maceración en el área y la presencia de orina y heces, a todos los cuidadores se les dieron indicaciones de cuidados generales del área del pañal para minimizar la humedad y la irritación, que fueron seguidos en prácticamente todos los casos.

En este grupo de lactantes casi una tercera parte había tenido en promedio dos episodios previos de dermatitis por pañal, a pesar de que $96 \%$ de los cuidadores referían un cambio de pañal en un lapso igual o menor a 4 horas; lo que podría indicar que otros factores irritativos no estaban adecuadamente controlados.

A lo largo del mes de seguimiento 20.6\% de los pacientes desarrolló dermatitis por pañal, lo cual es menor al 40 a $50 \%$ descrito por algunos autores ${ }^{6-9}$ y fue leve en más de $90 \%$ de los casos.

Es interesante que, preventivamente, BBTips ${ }^{\circledR}$ fueron más eficaces que Huggies UltraConfort ${ }^{\circledR}$ dado que se observó un porcentaje significativamente menor de casos de dermatitis por pañal en los lactantes que usaron BBTips ${ }^{\circledR}$. Ambos tipos de pañal tienen centro de celulosa y materiales absorbentes en gel, pero BBTips ${ }^{\circledR}$ además libera formulaciones por contacto, lo que podría explicar la mayor eficacia. Esta eficacia en la prevención de dermatitis por pañal fue corroborada por médicos y cuidadores.

La eficacia en el tratamiento de la se evaluó en lactantes con dermatitis por pañal (con o sin candidiasis agregada) al momento de la inclusión al estudio, con una muestra 5.8\% menor a la calculada (2 pacientes que no alcanzaron a ser incluidos y 2 pacientes con pérdida de seguimiento), que es menor al $10 \%$ esperado y considerado dentro de dicho cálculo. ${ }^{20}$
El tratamiento de la dermatitis por pañal irritativa consiste en disminuir la humedad en el área del pañal, minimizar el contacto de la piel con la orina y las heces y erradicar los microorganismos infecciosos. ${ }^{5}$ Para lograr dichos objetivos se debe enfatizar el aseo frecuente y cuidados de todos los posibles irritantes, con un limpiador que ayude a restaurar y mantener un $\mathrm{pH}$ fisiológico, seguido por secado completo y permitir que el área se oree. Debe revisarse si hay heces u orina cada 3 a 4 horas y realizar cambio de pañal con la mayor frecuencia posible. ${ }^{21}$ Finalmente se deben aplicar, en toda el área cubierta por el pañal, emolientes con función de barrera (que en su mayoría contienen óxido de zinc, vaselina sólida o una combinación de ambos) que ayuden a disminuir la fricción, prevenir la sobrehidratación y proveer protección contra las heces, la orina y otros irritantes y microorganismos. ${ }^{3,5}$ Por ello, a todos los cuidadores se les dieron las indicaciones apropiadas y lo único que varió fue la pasta del pañal elegida a criterio del médico tratante. Las indicaciones fueron seguidas prácticamente en todos los casos y la desviación encontrada fue el uso ocasional de toallitas húmedas para el aseo del área.

En este grupo de pacientes cerca de 90\% había tenido, en promedio, 3 episodios previos de dermatitis por pañal, casi un $20 \%$ más que en los lactantes sin dermatitis por pañal. El 91\% de los cuidadores refirieron realizar el cambio de pañal en un lapso igual o menor a 4 horas, un $6 \%$ por debajo de lo observado en el grupo de lactantes sin dermatitis por pañal.

En la evaluación inicial de los pacientes la frecuencia de formas graves fue muy semejante a la referida por otros autores: 6 versus $5 \%$, mientras que las formas leves y moderadas fueron ligeramente más frecuentes en nuestro estudio: 65 versus $50 \%$ y 29 versus $20 \%$, respectivamente. ${ }^{10}$

En la evaluación inicial 15\% de los pacientes tuvo candidiasis agregada. Cuando esto sucede 
se deben agregar al tratamiento antimicóticos tópicos como nistatina, o algún imidazol 2 o 3 veces al día. El uso de nistatina oral para eliminar Cándida del intestino, en combinación con nistatina tópica no ha mostrado ser más eficaz que el uso exclusivo de la forma tópica. ${ }^{24,25}$ Los pacientes con candidiasis recibieron el antimicótico tópico elegido por su médico tratante.

A la mitad del estudio se habían resuelto más de $50 \%$ de las dermatitis por pañal y las formas que persistieron fueron en su mayoría leves. Así mismo, la candidiasis disminuyó a una tercera parte. Al final del estudio se resolvieron $88 \%$ de las dermatitis por pañal y aquéllas que persistieron fueron en su mayoría leves, no hubo ningún caso de candidiasis.

Aunque un porcentaje elevado alcanzó la curación de la dermatitis por pañal a lo largo del estudio, es importante tratar de determinar porqué $12.5 \%$ de los pacientes aún cursaba con una forma leve de dermatitis por pañal. En casos de afección persistente es imperativo evaluar que todos los factores irritativos sean adecuadamente evaluados y resueltos, ${ }^{24}$ y también considerar algunos diagnósticos diferenciales de la dermatitis por pañal irritativa como psoriasis, acrodermatitis enteropática, hongos diferentes a Cándida e incluso histiocitosis de células de Langerhans. ${ }^{26}$

La eficacia del tratamiento de la dermatitis por pañal fue semejante para ambos tipos de pañal, a diferencia de lo ocurrido en la prevención donde BBTips $^{\circledR}$ fue superior. Sin embargo, la meta es disminuir la incidencia de dermatitis por pañal en los lactantes en riesgo, por lo que la prevención es uno de los aspectos más importantes a considerar.

Tanto en el aspecto preventivo como en el tratamiento de la dermatitis por pañal un elevado porcentaje de cuidadores volvería a utilizar los pañales prescritos. Esto pone de manifiesto que el uso de pañales desechables no sólo es eficaz, sino también seguro y que, en términos generales, los pañales con materiales absorbentes se asocian con formas clínicas menos graves de dermatitis por pañal que los pañales convencionales y los pañales de tela; ;2,14,15 por último, que los cuidadores son capaces de reconocer estas ventajas.

En general, existen estudios ${ }^{12-16,18}$ sobre la eficacia de los pañales para la prevención de dermatitis por pañal, incluso evaluada mediante revisión sistemática, ${ }^{27}$ que ponen de manifiesto que los pañales con centro de celulosa son superiores a los de tela; los pañales con materiales absorbentes en gel son superiores a los pañales con centro de celulosa exclusivamente, y que los pañales con liberación de formulaciones por contacto son superiores a aquéllos que no las liberan. El

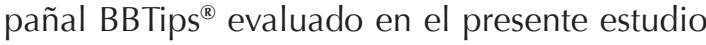
pertenece al grupo de liberación por contacto y, a pesar de ser semejante a Huggies UltraConfort ${ }^{\mathbb{B}}$ por pertenecer al grupo de absorbentes con gel, la superioridad global de BBTips ${ }^{\circledR}$ puede estar en relación con esta diferencia.

Por otro lado, no existen estudios que comparen a los diversos tipos de pañales cuando ya existe la dermatitis por pañal, por lo cual el presente es un estudio novedoso que pone de manifiesto que en el manejo de la dermatitis por pañal, además del tipo de pañal utilizado es muy importante el tratamiento agregado y el apego al mismo.

La dermatitis por pañal causa incomodidad en los niños, ansiedad en los padres y cuidadores y contribuye a la carga en el sistema de salud; existe una gran variedad de pañales en el mercado por lo que se requiere evidencia como la proporcionada en este estudio que ayude a tomar decisiones informadas para evaluar los pros y los contras de la elección de diferentes tipos de pañal. 


\section{Sesgos y limitaciones del estudio}

En el presente estudio no se tomaron en cuenta la lactancia ni el tipo de alimentación como variables a estudiar en ninguno de los grupos. Sin embargo, a través de la aleatorización, que se considera como el método electivo ideal para alcanzar la comparación entre los grupos, que en este estudio además se realizó mediante bloques balanceados, se consideró que dichas variables podrían estar distribuidas de forma representativa en los grupos estudiados. Otra limitación del estudio fue que el tiempo de seguimiento fue de tan sólo un mes y cabe la posibilidad que pasado ese tiempo se pudieran presentar nuevos casos de dermatitis por pañal.

\section{REFERENCIAS}

1. Odio M, Friedlander SF. Diaper dermatitis and advances in diaper technology. Curr Opin Pediatr. 2000;12(4):342-6.

2. Berg RW. Etiology and pathophysiology of diaper dermatitis. Adv Dermatol. 1988;3:75-90.

3. Adam R. Skin care of the diaper area. Pediatr Dermatol. 2008;25:427-33.

4. Berg RW. Etiology and pathophysiology of diaper dermatitis. Adv Dermatol. 1988;3:75-98.

5. Atherton DJ. The aetiology and management of irritant diaper dermatitis. J Euro Acad Dermatol Venereol. 2001;15(Suppl):1-4.

6. $\mathrm{Li} \mathrm{CH}, \mathrm{Zhu} \mathrm{ZH}$, Dai YH. Diaper dermatitis: a survey of risk factors for children aged 1-24 months in China. Int Med Res. 2012;40(5):1752-60.

7. Adalat S, Wall D, Goodyear H. Diaper dermatitis-frequency and contributory factors in hospital attending children. Pediatr Dermatol. 2007;24(5):483-8.

8. Philipp R, Hughes A, Golding J. Getting to the bottom of nappy rash. ALSPAC Survey Team. Avon Longitudinal Study of Pregnancy and Childhood.Br J Gen Pract. 1997;47(421):493-7.

9. Jordan WE, Lawson KD, Berg RW, Franxman JJ, Marrer AM. Diaper dermatitis: frequency and severity among a general infant population. Pediatr Dermatol. 1986;3(3):198-207.

10. Langoen A, Vik H, Nyfors A. Tidsskr Nor Naegeforen. Diaper dermatitis. Classification, occurrence, causes, prevention and treatment. 2003;113(14):1712-5.

11. Levin S. History of medicine-diapers. S Afr Med J. 1970;44:256-63.
12. Campbell RL. Clinical tests with improved disposable diapers. Pediatrician. 1987;14Suppl 1:34-8.

13. Lane AT, Rehder PA, Helm K. Evaluation of diapers containing absorbent gelling material with conventional disposable diapers in newborn infants. Am J Dis Child. 1990;144:315-8.

14. Odio MR, O'Connor RJ, Sarbaugh F, Baldwin SC. Continuous topical administration of a petrolatum formulation by a novel disposable diaper. Effect on skin surface microtopography. Pediatric Dermatol. 2000;200:232-7.

15. Erasala GN, Merlay I. Romain G. Evolution of disposable diapers and reduction of diaper dermatitis. Arch Pediatr. 2007;14(5):495-500.

16. Baldwin S, Odio MR, Haines SL, O'Connor RJ, Englehart JS, Lane AT. Skin benefits from continuous topical administration of a zinc oxide/petrolatum formulation by a novel disposable diaper. J Eur Acad Dermatol Venereol. 2001;15(Suppl 1):5-11.

17. Panahi Y, Sharif MR, Sharif A, Beiraghdar F, Zahiri Z. A randomized comparative trial on the therapeutic efficacy of topical aloe vera and calendula officinalis on diaper dermatitis in children. Scientifc World Journal 2012. Article ID 810234, 5 pages doi:10.1100/2012/810234.

18. Davis JA, Leyden JJ, Grove GL, Raynor WJ. Comparison of disposable diapers with fluff absorbent and fluff plus absorbent polymers: Effects on skin hydration, skin $\mathrm{pH}$, and diaper dermatitis. Pediatric Dermatol. 1989;6(2):102-8.

19. Estudio de Calidad. Pañales Desechables. La Misión Antiescurrimiento en Revista del Consumidor. 2011. http:www.revistadelconsumidor.gob.mx/wp-content/ uploads/2011/10/estudio-panales.pdf.

20. Lwanga SK, Lemeshow S. Sample size determination in health studies. A practical manual. Geneva: World Health Organisation, 1991.

21. Scheinfeld N. Diaper dermatitis: A review and brief survey of eruptions of the diaper area. Am J Clin Dermatol 2005;6:273-81.

22. Ward DB, Fleischer AB Jr, Feldman SR, Krowchuk DP. Characterization of diaper dermatitis in the United States. Arch Pediatr Adolesc Med 2000;154(9):943-6.

23. Bound JP. Thrush napkin rashes. BMJ 1956;1:782-4.

24. Shin HT. Diaper dermatitis that does not quit. Dermatol Therapy 2005;18:124-35.

25. Munz D, Powell KR, Pai CH. Treatment of candida diaper dermatitis: a double-blind placebo-controlled comparison of topical nystatin with topical plus oral nystatin. J Pediatr. 1982;101:1022-5.

26. Koblenzer PJ. Diaper dermatitis: an overview. ClinPediatr 1973;12:386-92.

27. Baer EL, Davies MW, Easterbrook KJ. Disposable nappies for preventing napkin dermatitis in infants. Cochrane Database Syst Rev. 2006; 19;(3):CD004262. 